Results Mean age was 75.3 (SD: 8.4) years old. 97 participants (75\%) were female. Mean BMI was $22.9 \mathrm{~kg} / \mathrm{m}^{2}$ (SD: 2.7, range: 17.8 to 30.9 ) which strongly correlated with waistline circumference $\left(\mathrm{r}^{2} 0.65, \mathrm{p}<0.0001\right)$ as shown in figure 1 (IDDF2021-ABS-0118 Figure 1). 40 participants (31\%) had NAFLD detected by abdominal ultrasonography. 35 (27\%) mild and 5 (4\%) moderate to severe. Individuals with NAFLD had significantly higher BMI, waist circumference, ALT, $\gamma$-GTP, TG and HbA1c levels compared to those without NAFLD. HDL levels were also significantly lower in NAFLD individuals. After adjusting for age, gender, TG and HbA1c levels, a higher BMI was significantly associated with the presence of NAFLD (adjusted OR 1.38, 95\%CI: 1.17 to 1.64 ).

Conclusions NAFLD was present in about $31 \%$ of non-drinking adults in this cohort, although most were classified as mild. As expected, a higher BMI was independently associated with the presence of NAFLD.

\section{IDDF2021-ABS-0141 PREDICTION OF MORTALITY AMONG ACUTE-ON-CHRONIC LIVER FAILURE PATIENTS USING ARTIFICIAL NEURAL NETWORK ANALYSIS}

${ }^{1}$ Balaji Musunuri*, 'Shiran Shetty, ${ }^{2}$ Dasharathraj K Shetty. ${ }^{1}$ Department of Gastroenterology, Kasturba Medical College, Manipal, Manipal Academy of Higher Education, Karnataka, India; ${ }^{2}$ Department of Humanities and Management, Manipal Institute of Technology, Manipal Academy of Higher Education, Karnataka, India

\subsection{6/gutjnl-2021-IDDF.94}

Background Despite the advances in understanding the pathophysiology and management of Acute-on-Chronic liver failure (ACLF), short-term mortality remains high. Though there have been numerous scoring systems to predict mortality, they lack predictive accuracy. We intend to study the accuracy of artificial neural network (ANN) analysis in predicting short mortality among such patients.

Methods Patients who were diagnosed as ACLF according to APASL criteria were included. Retrospective data of ACLF patients were collected, including the presence of decompensating events including ascites, encephalopathy, Gastrointestinal (GI) bleed, acute kidney injury (AKI) and infections. Basic blood investigations were recorded, including various prognostic scoring systems like CTP, MELD, CLIF-SOFA and AARC scores. A multi-layered perceptron ANN model with hidden nodes to make a prediction was constructed from 27 clinical and laboratory variables. The ANN was trained and validated internally using an adaptive moment estimation optimization algorithm. The primary endpoints were 30-day mortality.

Results One hundred patients were included with mean age of $46.04 \pm 11.28$ yrs. $88.4 \%$ were male. Most common acute precipitant of ACLF was alcohol followed by infections among $59.82 \%$ and $25 \%$ of patients respectively. DILI as acute cause was seen in $16.07 \%$, while $7.1 \%$ had unknown cause. Commonest etiology of CLD was alcohol followed by viral among $77.27 \%$ and $7.95 \%$ of patients. $73.2 \%$ had overt HE. GI Bleed, AKI and infections were noted in $19.6 \%, 46.4 \%$ and $32.1 \%$ respectively. Mortality at the end of 30-days was $36.6 \%$. Presence of AKI and GI bleed were significantly associated with mortality $(\mathrm{p}<0.05)$. Baseline CLIF, AARC, CTP, MELD had area under ROC of $0.704,0.767,0.652$ and 0.739 respectively in predicting 30-day mortality, while ANN had AUROC OF 0.915, having predictive accuracy of $94 \%$ outperforming the other prognostic scores.

Conclusions The artificial neural network had better accuracy in predicting short-term mortality among patients with ACLF. It has clinical utility in the management of ACLF, requiring further studies to validate its role.

\section{IDDF2021-ABS-0143 GLOBAL EPIDEMIOLOGICAL TRENDS OF FUNGAL INFECTIONS IN CIRRHOSIS PATIENTS: A SYSTEMATIC REVIEW WITH META-ANALYSIS (FUNGDEMIC)}

Nipun Verma*, Shreya Singh, Manvi Singh, Anil Chauhan, Pranita Pradhan, Nishant Jaiswal, Arunaloke Chakrabarti, Meenu Singh. Postgraduate Institute of Medical Education and Research, Chandigarh, India

\subsection{6/gutjnl-2021-IDDF.95}

Background Fungal infections (FIs) have serious implications, yet are poorly reported in cirrhosis patients. Therefore, we reviewed the global burden and trends of FIs among cirrhosis patients.

Methods PubMed, Ovid, Web of Science, and EMBASE were searched and full-text articles describing FIs and their prevalence among cirrhosis patients were reviewed. Studies from post-transplant, malignancy, and classical-immuno-deficiency patients were excluded. A random-effects meta-analysis was done to pool estimates of FIs (overall, and by mycological
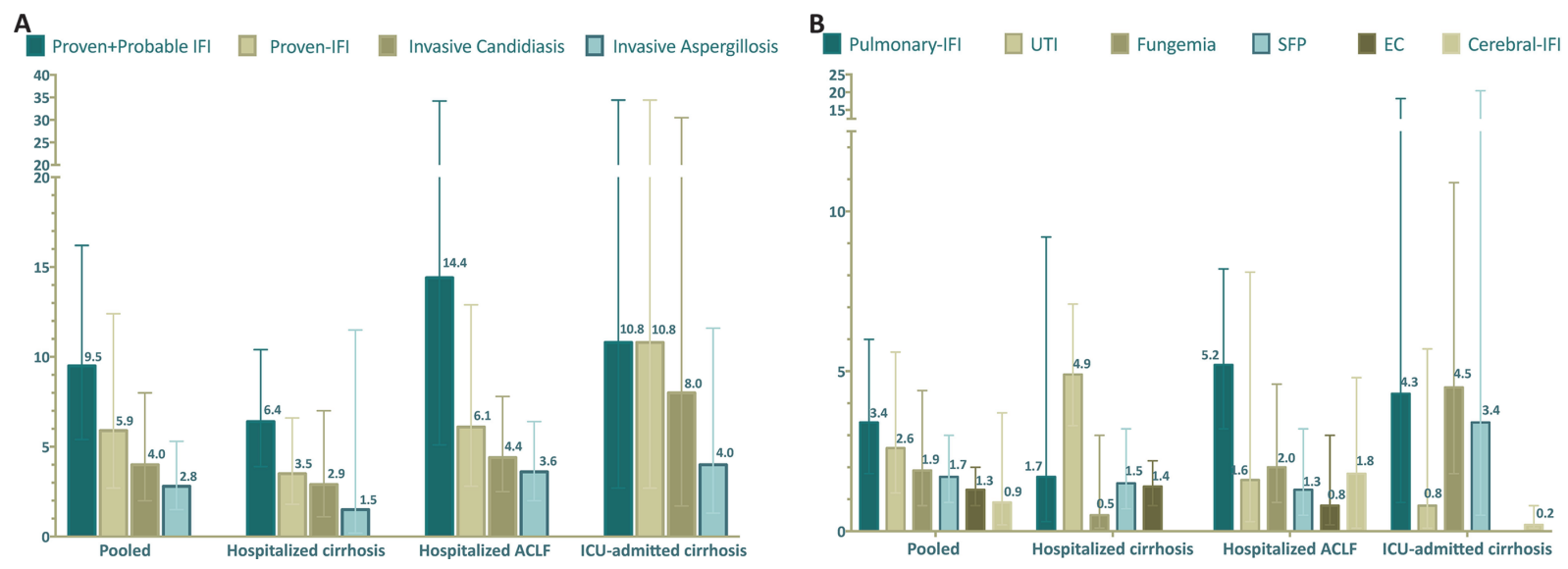

Abstract IDDF2021-ABS-0143 Figure 1 